\title{
Erratum: Lepton flavor violation in type I + III seesaw
}

\author{
Jernej F. Kamenik ${ }^{a, c}$ and Miha Nemevšek ${ }^{b, c}$ \\ ${ }^{a}$ INFN, Laboratori Nazionali di Frascati, \\ I-00044 Frascati, Italy \\ ${ }^{d}$ II. Institute für Theoretische Physik, Universität Hamburg, \\ Lurper Chaussee 149, 22761 Hamburg, Germany \\ ${ }^{c} J$. Stefan Institute, \\ 1000 Ljubljana, Slovenia \\ E-mail: jernej.kamenik@lnf.infn.it, miha.nemevsek@desy.de
}

ERRATUM TO: JHEP11(2009)023

ARXIV EPRINT: 0908.3451

On page 5 , there is a misprint in equations (3.2), (3.3) which should correctly read

$$
\begin{aligned}
& \tilde{g}_{L V}^{(p)}=2\left(1-4 s_{w}^{2}\right) L_{12}^{Z}, \\
& \tilde{g}_{R V}^{(p)}=2\left(1-4 s_{w}^{2}\right) R_{12}^{Z}, \\
& \tilde{g}_{L V}^{(n)}=-2 L_{12}^{Z}, \\
& \tilde{g}_{R V}^{(n)}=-2 R_{12}^{Z} \text {. }
\end{aligned}
$$

Accordingly, eq. (3.4) is modified to

$$
\left|L_{12}^{Z}\right|^{2}+\left|R_{12}^{Z}\right|^{2}<2.8 \times 10^{-13}, 2.3 \times 10^{-14},
$$

and the last sentence in the paragraph after equation (3.4) now reads as follows: "After allowing to vary the poorly known neutrino mass parameter $\theta_{13}$ within the allowed range in table 1 and the unknown phases $\delta$ and $\phi$, we obtain in the minimal models a bound on $\operatorname{Im}(z)<8.3(7.9)$ for normal (inverted) hierarchy in case of one triplet and one singlet and $\operatorname{Im}(z)<8.0(7.6)$ for two triplets, all at the reference mass of $m_{T}=100 \mathrm{GeV}$ for the lightest triplet."

Appropriate changes affect also figure 1, which is correctly given below.

The change also affects the last sentence in section 5 on page 9 , which should read as follows: "For example, if light neutrinos are degenerate with the sum of their masses close to the upper limit from $\beta$ decay and cosmology (say $\sum m_{\nu} \lesssim \mathrm{eV}[1]$ ), present $\mu-e$ conversion bounds already probe values of $\operatorname{Im}\left(z_{i}\right) \simeq 3-5$.".

Also, the third and fourth sentence in section 6 on page 9 now read: "Such a sensitivity would constrain $\operatorname{Im}(z)$ to 5.0 (4.6) in case of the minimal I + III model and to 4.6 (4.2) for the minimal type III, again for normal (inverted) hierarchy. For non-minimal models with degenerate $\mathrm{eV}$ scale neutrinos, these experiments would already probe $\operatorname{Im}\left(z_{i}\right) \simeq 1-2$ ".

Finally, we give the corrected figure 2 below. 

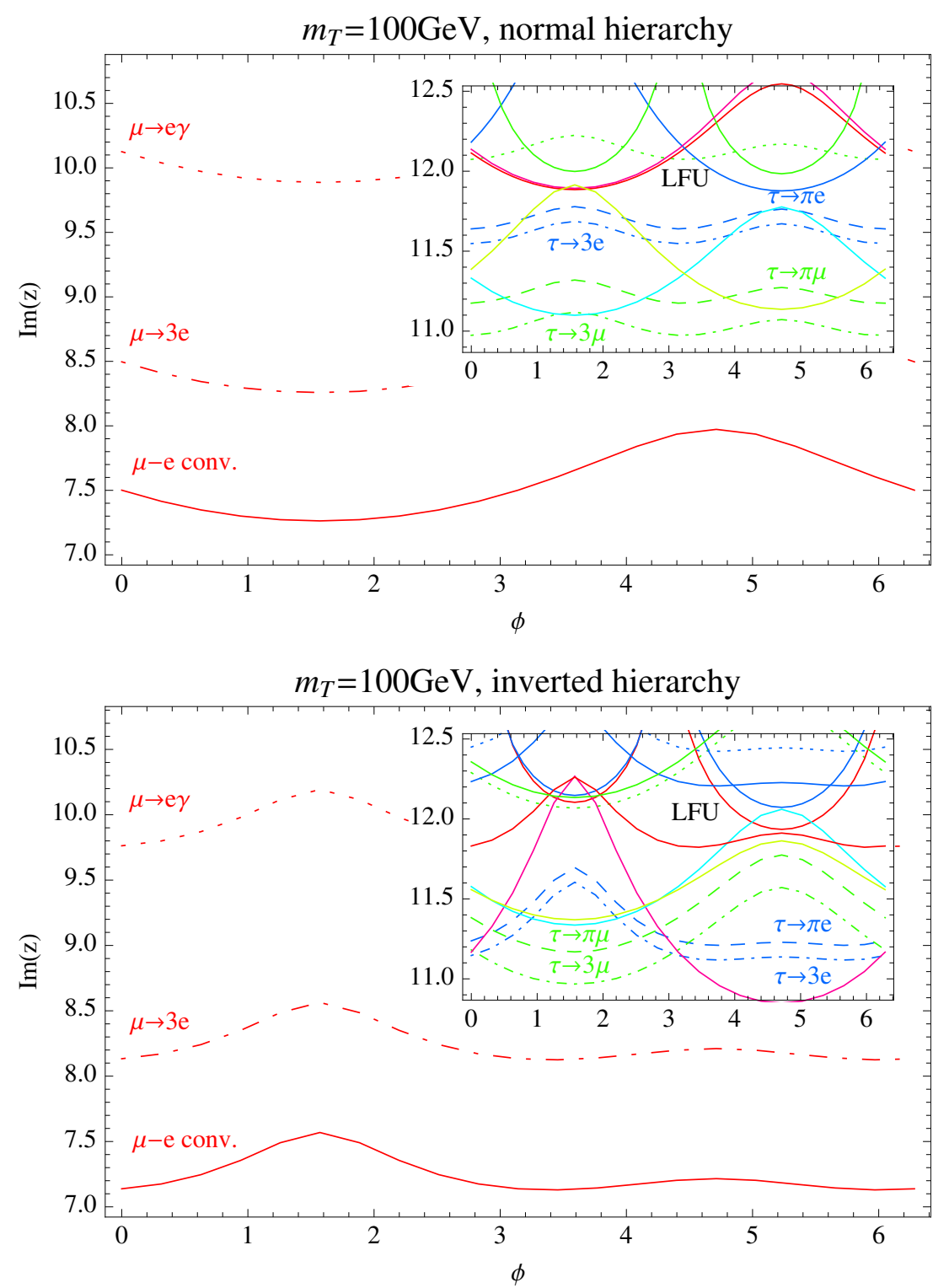

Figure 1. Comparison of various LFV and LFU bounds on the minimal type III model for normal (top) and inverted (bottom) neutrino mass hierarchy. The bounds coming from $\mu-e$ transitions are plotted in red, $\tau-e$ in blue and $\tau-\mu$ in green. Constraint from the $Z$ width to electrons is shown in magenta, to muons in cyan and to taus in yellow. The bounds constrain $\operatorname{Im}(z)$ at the reference triplets' mass of $100 \mathrm{GeV}$ and depend on the unknown Majorana phase $\phi$. Dependence on the other poorly known neutrino parameters is negligible as explained in the text. 


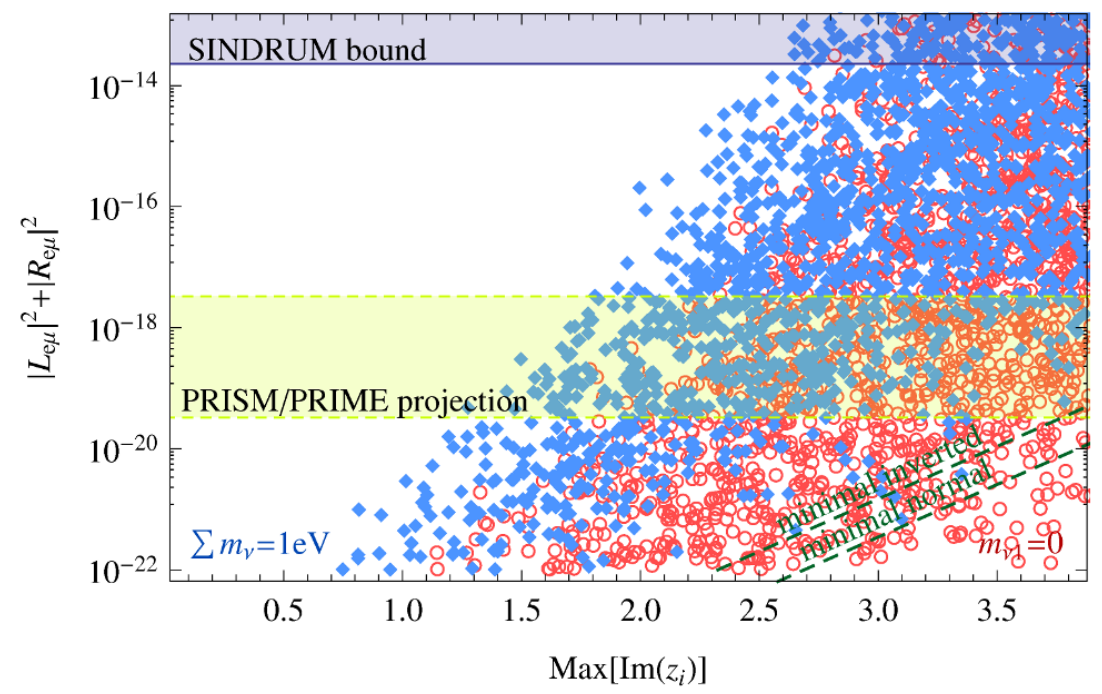

Figure 2. Present and projected sensitivity of $\mu-e$ conversion experiments in non-minimal type III see-saw models for a massless lightest neutrino (in red empty circles, for both hierarchies) and for degenerate scenario at $1 \mathrm{eV}$ (in blue filled spades). Minimal model predictions are drawn in green dashed lines. In all cases we put all the Majorana phases to zero and vary $z_{i}$ randomly.

Open Access. This article is distributed under the terms of the Creative Commons Attribution License (CC-BY 4.0), which permits any use, distribution and reproduction in any medium, provided the original author(s) and source are credited.

\section{References}

[1] A. Strumia and F. Vissani, Neutrino masses and mixings and..., hep-ph/0606054 [INSPIRE]. 